Research Paper

\title{
The Relation of the Level of Serum Anti-TF, -Tn and -Alpha-Gal IgG to Survival in Gastrointestinal Cancer Patients
}

\author{
Eugeniy Smorodin ${ }^{1}$, Boris Sergeyev ${ }^{1}$, Kersti Klaamasㄹ, Valentin Chuzmarov², Oleg Kurtenkov ${ }^{1}$ \\ 1. Department of Oncology \& Immunology, National Institute for Health Development, Tallinn 11619, Estonia. \\ 2. Department of Oncology \& II Surgery, North-Estonian Medical Centre, Tallinn 13419, Estonia.
}

$\triangle$ Corresponding author: Eugeniy Smorodin, PhD, Department of Oncology \& Immunology, National Institute for Health Development, Hiiu 42, 11619 Tallinn, Estonia. Tel., +372-6593934, Fax, +372-6593901, E-mail (can be published), jevgeni.smorodin@tai.ee.

(c) Ivyspring International Publisher. This is an open-access article distributed under the terms of the Creative Commons License (http://creativecommons.org/ licenses/by-nc-nd/3.0/). Reproduction is permitted for personal, noncommercial use, provided that the article is in whole, unmodified, and properly cited.

Received: 2013.06.06; Accepted: 2013.08.16; Published: 2013.09.23

\begin{abstract}
Objective: To evaluate the relation of the level of serum anti-TF, -Tn and $-\alpha$ Gal carbohydrate antibodies to survival in gastrointestinal cancer patients.

Methods: The level of anti-TF (Thomsen-Friedenreich antigen), $-T n$ and $-\alpha G a l$ lgG was analysed in the serum of patients with gastric $(n=83)$ and colorectal $(n=5 I)$ cancers in the long-term follow-up, using ELISA with polyacrylamide glycoconjugates. To evaluate overall survival and the risk of death, the Kaplan-Meier method and the Cox proportional hazards model were used in the univariate analysis of patients groups.

Results: A significantly better survival was observed: (I) in patients with an increased level of anti-TF antibodies (all, stage III, T2-4, NI-2 and G3; P = 0.004-0.038, HR =0.16-0.46); and (2) in patients with an increased level of anti-Tn antibodies (GI-2 tumors; $P=0.034-0.042, H R=$ $0.34-0.47)$. A significantly worse survival was observed in gastrointestinal, gastric and colorectal groups with an increased level of serum anti- $\alpha \mathrm{Gal}$ antibodies. This association depended on the patho-morphology of tumors (all, stages I-II, III, T2-4, NO, NI-2 and GI-2; P = 0.006-0.048, HR = I.99-2.33). In the combined assessment of the anti-TF and $-\alpha \mathrm{Gal}$ antibodies level of the whole gastrointestinal group $(n=53), P=0.002, H R=0.25,95 \% \mathrm{Cl} 0.094-0.655$. In the follow-up, the survival time was shorter in patients whose level of anti- $\alpha \mathrm{Gal}$ antibodies rose $(P=0.009-0.040$, $H R=2.18-4.27)$. The level of anti-TF antibodies inversely correlated with neutrophil/lymphocyte ratio (NLR, $r=-0.40 \mathrm{I}, \mathrm{P}=0.004, n=49)$. Patients with a higher level of anti- $\alpha \mathrm{Gal}$ antibodies and NLR values demonstrated a significantly worse survival $(P=0.009, H R=2.98, n=48)$.
\end{abstract}

Conclusions: The preoperative levels of anti-TF, $-\mathrm{Tn}$ and $-\alpha \mathrm{Gal}$ antibodies and their dynamics are of prognostic significance. The method for the determination of circulating anti-carbohydrate antibodies may be a useful supplement in clinical outcome assessment.

Key words: gastrointestinal cancer, survival, Thomsen-Friedenreich, Tn, alpha-Gal, antibodies, enteric microbiota.

\section{Introduction}

Anti-carbohydrate antibodies in human are constantly stimulated by enteric microbiota. In different pathologies of the digestive tract, including cancer, the altered colonization and bacterial translocation occurr [1-3] that may change the pattern and level of circulating anti-carbohydrate antibodies. Comparative data about spontaneously occurring anti-carbohydrate antibodies in relation to survival of 
patients with gastrointestinal cancer are scanty, but may be of clinical significance. The Thomsen-Friedenreich antigen (TF, Galß1-3GalNAca) and its precursor Tn (GalNAca) belong to the mucin-type tumor-associated carbohydrate antigens (TACA). They are usually cryptic epitopes in normal tissues but frequently expressed in adenocarcinomas $[4,5]$. The increased expression of TF and Tn antigens is associated with the tumor invasion and metastases [4, 6]. The $\alpha$ Gal epitope (Gal $\alpha 1-3 \mathrm{Gal} \beta 1-4 \mathrm{GlcNAc}$ ) is also cryptic and is only aberrantly expressed on human cells [7]. It is considered that the natural anti-TF, -Tn and $-\alpha$ Gal antibodies in human are induced by gastrointestinal microorganisms $[7,8]$. We used synthetic homogeneous polyacrylamide conjugates [9], viz. TF-pAA, Tn-PAA and $\mathrm{B}_{\mathrm{di}}-\mathrm{PAA}\left(\mathrm{B}_{\mathrm{di}}\right.$ is a minimal ligand of anti- $\alpha \mathrm{Gal}$ antibodies) to determine antibody levels in the sera of donors and patients with cancer. The preoperative level of anti-TF antibodies was significantly decreased in the serum of patients $[10,11]$. This is in accordance with the results obtained by other researchers who used the erythrocyte-derived TF-antigen in the assay of anti-TF antibodies [12]. The elevated postoperative levels of anti-TF, -Tn and $-\alpha \mathrm{Gal}$ IgG observed in the follow-up were associated with the stage and morphology of the tumor [13]. The anti- $\alpha \mathrm{Gal}$ antibodies may be involved in the progression of autoimmune diseases [7] but have been studied insufficiently with reference to cancer.

In the present study, the preoperative level of anti-TF, -Tn and $-\alpha \mathrm{Gal}$ IgG and its changes in the follow-up were investigated in relation to survival. Also stages of the disease, tumor size status, morphology, tumor spread in regional lymph nodes and hematological parameters were considered.

\section{Material and Methods}

\section{Patients}

The investigation was carried out in accordance with the ICH GCP Standards and approved by Tallinn Medical Research Ethics Committee, Estonia. The informed consent was obtained from each patient under examination. Altogether two hundred nineteen patients with gastrointestinal cancer, whose diagnosis was verified by the PTNM system [14], were investigated. More homogeneous groups were subjected to the long-term follow-up, excluding patients with distant metastases, those who underwent non-resective surgery, or individuals who during dynamic investigations survived less than three months after surgery. Also, patients who died of causes other than tumor recurrence were excluded from the study. The chemoand X-ray therapy influenced the circulating antibody level, therefore, therapy-prescribed patients were excluded. Survival was evaluated in selected patients (n = 134), among them ninety-nine underwent a long-term follow-up. The median and mean age \pm SD of males was 61 and $60.2 \pm 9.3(n=68)$, and of females 63 and $61.5 \pm 9.6(\mathrm{n}=66)$, respectively. The curative surgery, involving the extended D2 gastrectomy with lymphadenectomy and the resection of local lesions for colorectal cancer, was performed. Regional lymph node metastases were also removed in advanced cancer. The venous blood samples were taken before and after a surgical operation, and during the planned visits to the physician for health control according to standard protocol. Within the first 2 years after surgery, a follow-up every 3-6 months consisted of a clinical examination, routine blood testing, assessment of tumor markers concentration, abdominal ultrasonography, CT scanning and endoscopy. During the next years, the patients were followed up every 6-12 months.

\section{Glycoconjugates}

The synthetic glycoconjugates (Mr $30 \mathrm{kDa}$ ) were obtained from Lectinity (Russia). The soluble TF conjugate with N-unsubstituted polyacrylamide (TF-pAA, amide-type) as well as ethanolamide-type glycoconjugates of the poly[N-(2-hydroxyethyl) acrylamide] namely, Tn-PAA and $\alpha$ Gal-PAA $\left(B_{\text {di }}-P A A\right.$, in which $B_{\text {di }}$ is Gal $\left.\alpha 1-3 G a l \beta 1\right)$, were used in ELISA. The TF-pAA contained $0.1 \mathrm{~mol}$ of TF per $1 \mathrm{~mol}$ of pAA. The rest of the conjugates had $0.2 \mathrm{~mol}$ of a saccharide per $1 \mathrm{~mol}$ of PAA. Tris(hydroxymethyl) aminomethane-PAA was used as an adequate negative control [11].

\section{The indirect ELISA}

The assay was performed as described earlier [15]. The sera were collected by centrifugation of clotted venous blood after incubation for $2 \mathrm{~h}$ at $37^{\circ} \mathrm{C}$. The sera were kept at $4^{\circ} \mathrm{C}$ for no longer than three weeks or were frozen $\left(-50^{\circ} \mathrm{C}\right)$ and thawed once before use. The glycoconjugates with PAA $(5 \mu \mathrm{g} / \mathrm{ml})$ in 0.05 $\mathrm{M}$ carbonate buffer, $\mathrm{pH}$ 9.2, were applied to the Nunc-Immuno plate (MaxiSorp) and held overnight at $4{ }^{\circ} \mathrm{C}$. After washing with $0.05 \mathrm{M}$ Tris $\mathrm{HCl} / 0.2 \mathrm{M}$ $\mathrm{NaCl} / 0.02 \%$ sodium azide/0.05\% Tween 20, pH 7.5 (TBS), the plate was coated with human sera diluted $(1: 25$ - 1:200) in TBS/5 mM EDTA/0.25\% bovine serum albumin (BSA) and incubated for $2 \mathrm{~h}$ at $26^{\circ} \mathrm{C}$. The plate was washed with TBS and goat anti-human IgG-alkaline phosphatase conjugate in TBS was added. The plate was kept for $1.5 \mathrm{~h}$ at $26^{\circ} \mathrm{C}$ and washed. The absorbance (A) at $405 \mathrm{~nm}$ was measured using a Labsystem Multiscan MCC/340 (Finland) after incubation for $1 \mathrm{~h}$ at $26^{\circ} \mathrm{C}$ with a p-nitrophenylphosphate 
disodium salt $(1 \mathrm{mg} / \mathrm{ml}$ in $0.1 \mathrm{M}$ glycine-buffer, $\mathrm{pH}$ 10.3). Each sample was analyzed in dublicate, including analysis with Tris-PAA (negative control). The antibody level was estimated as a ratio of $\mathrm{A}_{\mathrm{t}}$ est $/ A_{\text {control, }}$ where $A_{\text {test }}$ is the absorbance with the PAA-glycoconjugate and $\mathrm{A}_{\text {control, }}$ with the Tris-PAA. The variation coefficient was $3 \%$.

\section{Clinical analysis of blood samples}

Biochemical and hematological analyses were performed in the Oncological Centre of the North-Estonian Regional Hospital, using a Hitachi 912 and Elecsys 2010, Roche Diagnostics; a Sysmex XE-2100, Sysmex Corporation. The antibody levels were correlated with the count and percentage of leukocytes, neutrophils, monocytes, lymphocytes and eosinophils, and neutrophile/lymphocyte ratio (count).

\section{Statistical analysis}

The level of antibodies and its dynamics were studied in relation to overall survival time starting from the date of the preoperatively taken blood sample. The Kaplan-Meier method was used for the estimation of survival curves in the univariate analysis of patients groups, the significance of differences was analyzed by the log-rank test. The Cox proportional hazards model was used to evaluate the risk of death. Statistical analysis was performed using SPSS, version 15.0. The median (M) of the level of anti-TF, -Tn and $-\alpha \mathrm{Gal}$ antibodies was used as cut-off to discriminate between responders ( $R$, the level above or equal to $M$ ) and weak responders (WR, the level below $M)$. Survival of $\mathrm{R}$ vs WR was evaluated in subgroups divided by stages, tumor volume, tumor grade and regional lymph node metastases. The difference in survival between patient groups was considered to be significant when $P \leq 0.05$. The linear regression analysis was conducted using a Statgraphics Plus 5.1. The data were approximated to a quadratic function, using a CurveFinder for CurveExpert (Version 1.34).

\section{Results}

\section{The relation of the preoperative antibody level to survival}

In general, the TF-R of different groups demonstrated a better survival $(\mathrm{HR}<1$, mean survival time of $R>W R$ ) while $\alpha$ Gal-R revealed a worse survival ( $H R>1$, mean survival time of $R<W R$, Table 1, Figure $1 \mathrm{~A}, \mathrm{C}$ ). In both gastrointestinal and gastric cancer, the significantly better survival in R vs WR was associated with tumor grade: G3 for TF-R and G1-2 for Tn-R (Table 1, Figure 1 B). The TF-R exhibited a significantly better survival in advanced gastrointestinal cancer in stage III, T2-4 and N1-2 (the difference in survival between cancer patients in stages I-II, T1 or N0 was insignificant). The $\alpha$ Gal-R showed a significantly worse survival in stages I-II and N0 in gastric cancer but in stage III and N1-2 in colorectal cancer. No significant differences in survival between TF-R and TF-WR, as well as between Tn-R and Tn-WR in coloractal cancer were observed. We did not observe the relation of survival to tumor morphology in patients with gastric, colorectal or gastrointestinal cancer (G1-2 vs G3, P = $0.24-0.54)$. Survival was not associated with the age and sex of patients either $(\mathrm{P}=$ $0.20, \mathrm{P}=0.65)$. Survival was better in $\mathrm{N} 0$ status ( $\mathrm{N} 0$ vs $\mathrm{N} 1-2, \mathrm{P}=0.046, \mathrm{n}=134, \mathrm{HR}=0.60,95 \%$ CI 1.01-2.76).

Patients selected from all gastrointestinal groups, whose level of anti-TF antibodies was increased and that of anti- $\alpha \mathrm{Gal}$ antibodies decreased, i.e. TF-R/ $\alpha$ Gal-WR, demonstrated a significantly better survival compared to patients TF-WR/ $\alpha$ Gal-R $(\mathrm{P}=$ $0.002, \mathrm{HR}=0.25,95 \% \mathrm{CI}$ 0.094-0.655, mean survival time 156.1 vs $91.9, \mathrm{n}=53$, Figure 2A).

\section{The relation of the antibody level dynamics to survival}

The level of IgG antibodies to glycoconjugates in the serum of patients changed after surgery [13]. We delineated the change of antibody level as a quadratic function $\mathbf{Y}=\mathbf{a}+\mathbf{b X}+\mathbf{c} \mathbf{X}^{2}$, where $\mathbf{Y}$ is the level of antibodies, $\mathbf{X}$ is the time in months, coefficient $\mathbf{a}$ is the factor characterizing the initial level of antibodies, $\mathbf{b}$ and $\mathbf{c}$ are the factors characterizing its dynamics ( $\mathbf{b}$ is the change of antibody level by month and $\mathbf{c}$ is the acceleration of antibody level change). This function showed a good fit to the data points ( $\mathrm{r}=0.84-0.95)$ and was applied to the calculation of factors for each patient. We did not examine the dependence of survival on factor $\mathbf{c}$ because of its insignificant influence on antibody level dynamics. The medians of factors a and $\mathbf{b}$ were used as cut-off to discriminate between responders (the value above or equal to $\mathrm{M}$ ) and weak responders (the value below $\mathrm{M}$ ). Evaluated by factor $\mathbf{b}$, the antibody levels in responders were prone to rise after surgery (positive values of the median and mean of factor $\mathbf{b}$ ), but the levels diminished in most weak responders (negative values of the mean factor $\mathbf{b}$, Table 2). 
Table I. The relation of the preoperative antibody level in the serum of cancer patients to survival.

\begin{tabular}{|c|c|c|c|c|c|c|c|}
\hline Antibodies & Group & Median (M) & $\mathrm{n}$ & $\mathrm{P}$ & HR & $95 \% \mathrm{CI}$ & Mean survival time, months * \\
\hline \multirow[t]{7}{*}{ TF } & Gastrointestinal, all & 1.17 & 106 & 0.013 & 0.46 & $0.24-0.86$ & $145.3 ; 104.3$ \\
\hline & Stage III & & 36 & 0.036 & 0.24 & $0.06-1.03$ & $122.4 ; 69.5$ \\
\hline & $\mathrm{T} 2-\mathrm{T} 4$ & & 85 & 0.011 & 0.41 & $0.20-0.84$ & $138.8 ; 88.4$ \\
\hline & N1-2 & & 39 & 0.038 & 0.24 & $0.06-1.04$ & $124.5 ; 74.2$ \\
\hline & G3 & & 48 & 0.004 & 0.16 & $0.04-0.67$ & $173.6 ; 99.5$ \\
\hline & Gastric, all & 1.14 & 66 & 0.075 & 0.51 & $0.24-1.08$ & $143.2 ; 103.5$ \\
\hline & G3 & & 39 & 0.016 & 0.30 & $0.11-0.85$ & $151.3 ; 88.9$ \\
\hline \multirow[t]{4}{*}{$\mathrm{Tn}$} & Gastrointestinal, all & 1.41 & 134 & 0.401 & & & \\
\hline & G1-2 & & 73 & 0.034 & 0.47 & $0.23-0.96$ & $125.5 ; 104.5$ \\
\hline & Gastric, all & 1.43 & 83 & 0.806 & & & \\
\hline & G1-2 & & 37 & 0.042 & 0.34 & 0.11-1.01 & $135.8 ; 106.3$ \\
\hline \multirow[t]{13}{*}{$\alpha \mathrm{Gal}$} & Gastrointestinal, all & 4.11 & 134 & 0.006 & 2.19 & $1.24-3.87$ & $104.5 ; 145.4$ \\
\hline & Stages I-II & & 85 & 0.048 & 2.20 & $0.99-4.91$ & $120.9 ; 156.2$ \\
\hline & Stage III & & 49 & 0.036 & 2.33 & $1.03-5.24$ & $74.5 ; 122.1$ \\
\hline & $\mathrm{T} 2-\mathrm{T} 4$ & & 106 & 0.020 & 1.99 & $1.10-3.59$ & $94.6 ; 135.2$ \\
\hline & No & & 78 & 0.056 & 2.28 & $1.02-5.08$ & $119.8 ; 153.3$ \\
\hline & N1-2 & & 56 & 0.070 & 2.08 & $0.93-4.69$ & $82.2 ; 124.7$ \\
\hline & G1-2 & & 73 & 0.048 & 2.03 & $0.99-4.16$ & $101.3 ; 135.0$ \\
\hline & G3 & & 61 & 0.051 & 2.49 & $0.96-6.45$ & $108.3 ; 152.1$ \\
\hline & Gastric, all & 4.34 & 83 & 0.106 & & & \\
\hline & I-II & & 54 & 0.039 & 3.14 & $1.00-9.89$ & $126.3 ; 170.2$ \\
\hline & N0 & & 50 & 0.031 & 3.30 & $1.05-10.37$ & $125.3 ; 169.5$ \\
\hline & Colorectal, all & 2.99 & 51 & 0.044 & 2.86 & $0.96-7.90$ & $89.5 ; 120.9$ \\
\hline & Stage III or N1-2 & & 23 & 0.024 & 7.85 & $0.97-65.59$ & $74.9 ; 142.8$ \\
\hline
\end{tabular}

" Responders vs weak responders.
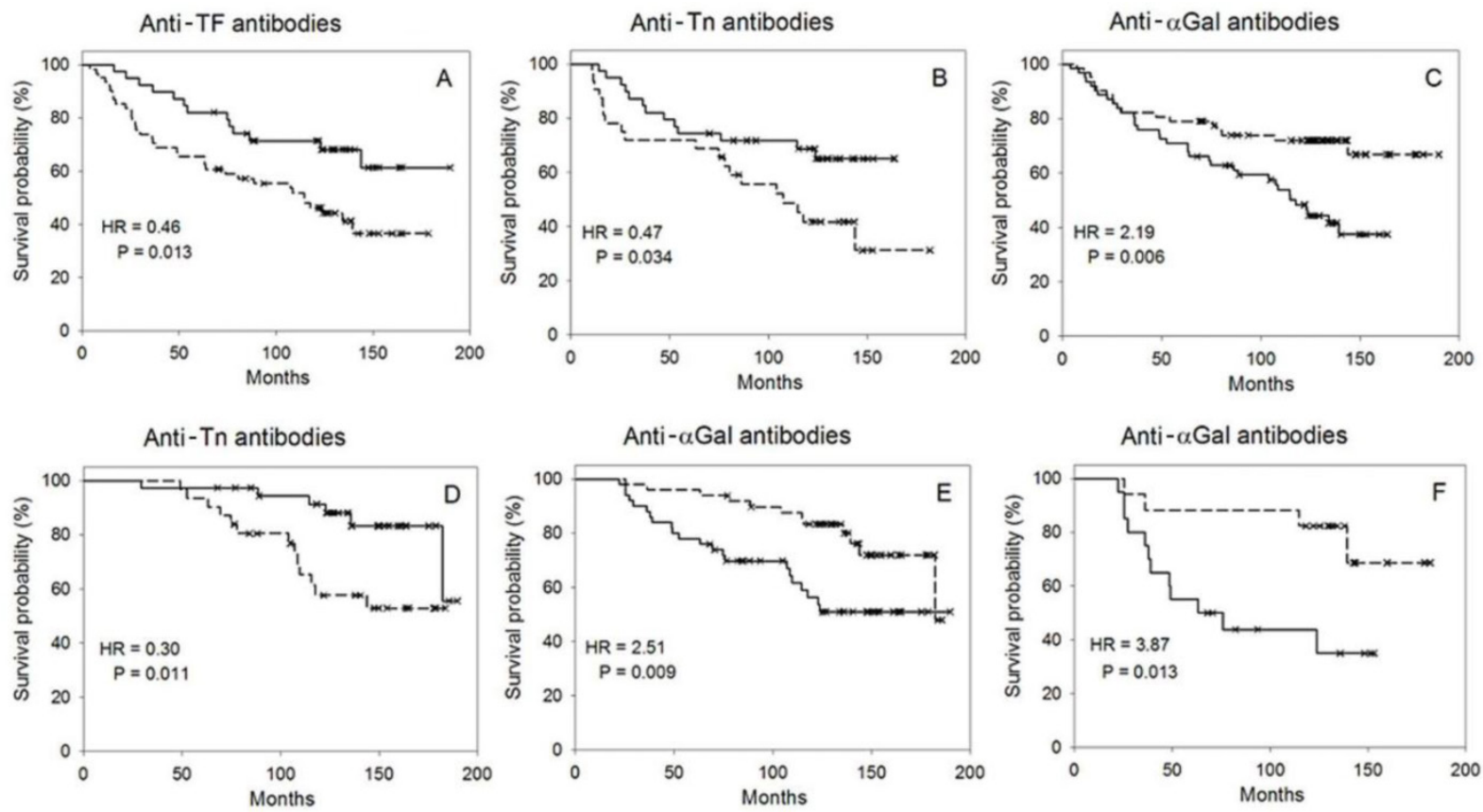

Fig I. The probability of survival in responders (the level of serum antibodies above or equal to median $\mathrm{M}$, a solid line) vs weak responders (the level below $\mathrm{M}$, a dotted line). A, B, C - the relation of the preoperative antibody level to survival; D, E, F - the relation of the antibody level dynamics to survival. A anti-TF antibodies, all patients with gastrointestinal cancer; B - anti-Tn antibodies, GI-2 tumors of gastrointestinal cancer; C - anti- $\alpha$ Gal antibodies, all patients with gastrointestinal cancer. D - anti-Tn antibodies, gastrointestinal cancer in stages I-II, the evaluation of survival by factor a. E, $F-$ anti- $\alpha \mathrm{Gal}$ antibodies, the evaluation of survival by factor b, E - all patients with gastrointestinal cancer, F - patients with an NI-2 status of gastrointestinal cancer (medians shown in Table 2). 
Table 2. Median and mean of factors a and b (the whole gastrointestinal group).

\begin{tabular}{|c|c|c|c|c|}
\hline Antibodies & Factor & Median (M) & Mean $\pm \mathrm{SD}^{*}$, responders & Mean \pm SD, weak responders \\
\hline \multirow[t]{2}{*}{ TF } & a & 1.130 & $2.153 \pm 1.903$ & $0.947 \pm 0.297$ \\
\hline & $\mathrm{b}$ & 0.001 & $0.039 \pm 0.071$ & $-0.011 \pm 0.017$ \\
\hline \multirow[t]{2}{*}{ Tn } & a & 1.490 & $3.292 \pm 2.189$ & $0.975 \pm 1.126$ \\
\hline & $\mathrm{b}$ & 0.0001 & $0.058 \pm 0.149$ & $-0.031 \pm 0.054$ \\
\hline \multirow[t]{2}{*}{$\alpha \mathrm{Gal}$} & $\mathrm{a}$ & 4.440 & $8.542 \pm 4.850$ & $2.144 \pm 2.134$ \\
\hline & $\mathrm{b}$ & 0.006 & $0.199 \pm 0.324$ & $-0.082 \pm 0.161$ \\
\hline
\end{tabular}

*SD - standard deviation
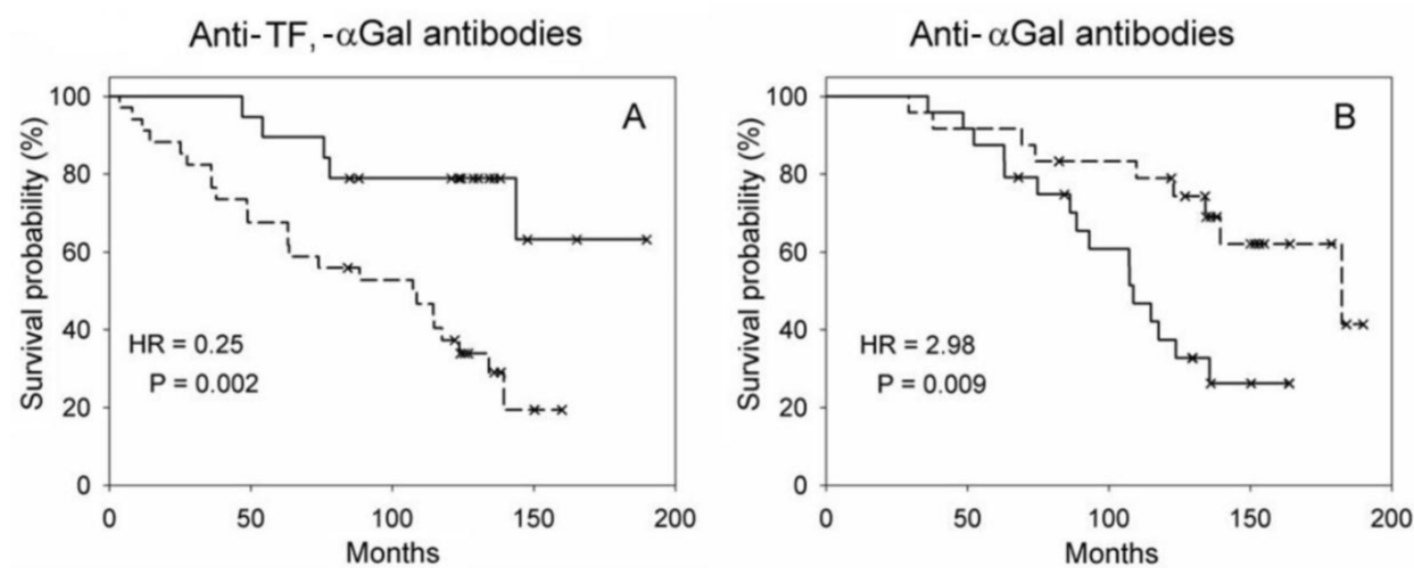

Fig 2. The probability of survival. A - TF responders/ $\alpha$ Gal weak responders (a solid line) vs TF weak responders/ $\alpha$ Gal responders (a dotted line) in the whole gastrointestinal group. $B-\alpha$ Gal responders having mean NLR $\geq M$ (a solid line) vs $\alpha$ Gal responders having mean NLR $<M$ (a dotted line).

Similar to the results of the preoperative assessment, survival of TF-R vs TF-WR evaluated by factor a was significantly better in the whole gastrointestinal group as well as in groups G3 and T2-4 (Table 3). Survival was not associated with the dynamics of anti-TF antibody level as evaluated by factor $\mathbf{b}$. The survival of Tn-R evaluated by factor $\mathbf{a}$ was better in stages I-II, T2-4, N0 and G1-2 (Table 3, Figure $1 \mathrm{D})$. No difference in survival evaluated by factor a of anti-TF, -Tn- and - $\alpha \mathrm{Gal}$ antibodies was found between patients in stage III or group N1-2. Evaluated by factor $\mathbf{b}$, the survival was found to be worse in the Tn-R of gastric cancer in stage III or N1-2. In stages I-II or group N0, the survival of $\alpha \mathrm{Gal}-\mathrm{R}$ evaluated by factor a was significantly worse. Also, the worse survival of $\alpha$ Gal-R evaluated by factor $\mathbf{b}$ was observed in different groups, including patients with and N1-2 status and not those with an N0 status (Table 3, Figure $1 \mathrm{E}, \mathrm{F})$.

\section{The relation of antibody levels to hematolog- ical parameters}

The neutrophil/lymphocyte ratio (NLR) reflects the inflammatory response. The NLR values of patients was changed in the follow-up. The mean value of NLR in each patient was calculated and the median of mean values was used as a cut-off criterion. Survival was worse in the whole group of patients with gastrointestinal cancer, whose mean NLR $\geq$ median $(\mathrm{M}=1.95, \mathrm{P}=0.059, \mathrm{n}=72, \mathrm{HR}=2.09,95 \% \mathrm{CI}$ $0.96-4.59)$. The $\alpha \mathrm{Gal}$ responders having mean NLR $\geq$ $M(M=1.90)$ demonstrated a significantly worse survival than $\alpha \mathrm{Gal}$ responders, whose mean NLR $<\mathrm{M}$ (P $=0.009, \mathrm{n}=48, \mathrm{HR}=2.98,95 \%$ CI 1.26-7.05, Figure 2B). The relationship between the level of anti- $\alpha \mathrm{Gal}$ antibodies and NLR was observed in the follow-up in some patients. Patients whose level of anti- $\alpha \mathrm{Gal}$ antibodies directly correlated with NLR $(\mathrm{r} \geq 0.38, \mathrm{n}=$ 28) showed a worse survival compared to patients, whose antibody level did not correlate or correlated inversely with NLR $(\mathrm{n}=25): \mathrm{P}=0.017, \mathrm{HR}=4.27,95 \%$ CI 1.17-15.52. The level of anti- $\alpha \mathrm{Gal}$ antibodies slightly correlated with the percentage of monocytes $(r=0.265, P=0.038, n=62)$. The level of anti-TF antibodies correlated with the count of lymphocytes ( $\mathrm{r}$ $=0.446, \mathrm{P}=0.001$ ) but correlated inversely with the count of neutrophils $(r=-0.300, P=0.036)$ and NLR ( $r$ $=-0.401, P=0.004, n=49$ in all cases, Figure 3$)$. The level of anti-Tn antibodies did not correlate with the count or percentage of neutrophils, lymphocytes and monocytes, and NLR. The level of anti-TF, -Tn and $-\alpha \mathrm{Gal}$ antibodies did not correlate with the count or percentage of leukocytes, platelets and eosinophils. No correlation between the age of patients and their serum antibody level was observed. 
Table 3. The relation of the antibody level dynamics to survival.

\begin{tabular}{|c|c|c|c|c|c|c|c|}
\hline Antibodies & Group & Factor & $\mathrm{n}$ & $\mathrm{P}$ & HR & $95 \%$ CI & Mean survival time, months * \\
\hline \multirow[t]{6}{*}{ TF } & Gastrointestinal, all & a & 99 & 0.035 & 0.48 & $0.24-0.96$ & $160.3 ; 134.3$ \\
\hline & $\mathrm{T} 2-4$ & & 81 & 0.042 & 0.47 & $0.22-0.98$ & \\
\hline & G3 & & 45 & 0.016 & 0.27 & $0.08-0.84$ & $169.5 ; 123.2$ \\
\hline & Gastric, all & $\mathbf{a}$ & 62 & 0.067 & & & $163.8 ; 129.2$ \\
\hline & $\mathrm{T} 2-4$ & & 45 & 0.034 & 0.34 & $0.12-0.97$ & \\
\hline & G3 & & 37 & 0.020 & 0.24 & $0.07-0.88$ & $172.8 ; 116.4$ \\
\hline \multirow[t]{16}{*}{ Tn } & Gastrointestinal, all & $\mathbf{a}$ & 99 & 0.052 & 0.51 & $0.25-1.02$ & $158.9 ; 134.3$ \\
\hline & Stages I-II & & 68 & 0.011 & 0.30 & $0.11-0.80$ & $173.5 ; 141.9$ \\
\hline & $\mathrm{T} 2-4$ & & 81 & 0.036 & 0.46 & $0.22-0.97$ & $155.1 ; 125.6$ \\
\hline & No & & 62 & 0.038 & 0.37 & $0.14-0.98$ & $171.7 ; 145.0$ \\
\hline & G1-2 & & 54 & 0.041 & 0.39 & $0.15-0.99$ & $152.1 ; 128.9$ \\
\hline & T2-4N0 & & 47 & 0.010 & 0.24 & $0.08-0.78$ & $157.9 ; 116.4$ \\
\hline & T2-4N1-2 & & 34 & 0.79 & & & \\
\hline & Gastric, all & $\mathbf{a}$ & 62 & 0.049 & 0.41 & $0.17-1.02$ & $163.5 ; 130.3$ \\
\hline & Stages I-II & & 44 & 0.041 & 0.27 & $0.07-1.04$ & $178.6 ; 146.6$ \\
\hline & $\mathrm{T} 2-4$ & & 45 & 0.049 & 0.39 & $0.15-1.03$ & $157.9 ; 116.4$ \\
\hline & G1-2 & & 25 & 0.056 & 0.24 & $0.05-1.18$ & \\
\hline & Stage III & $\mathbf{b}$ & 18 & 0.044 & 4.41 & $0.92-21.14$ & $68.6 ; 133.6$ \\
\hline & $\mathrm{N} 1-2$ & & 24 & 0.016 & 4.57 & $1.18-17.61$ & $72.6 ; 142.0$ \\
\hline & Colorectal, all or T2-4 & $\mathbf{a}$ & 37 & 0.024 & 0.24 & $0.06-0.91$ & $169.4 ; 122.1$ \\
\hline & Stages I-II or N0 & & 23 & 0.025 & 0.19 & $0.04-0.95$ & $175.6 ; 115.8$ \\
\hline & G1-2 & & 29 & 0.010 & 0.17 & $0.04-0.78$ & \\
\hline \multirow[t]{19}{*}{$\alpha \mathrm{Gal}$} & Gastrointestinal, all & $\mathbf{a}$ & 99 & 0.056 & 1.96 & $0.97-3.96$ & $136.5 ; 159.1$ \\
\hline & Stages I-II & & 68 & 0.030 & 2.90 & $1.05-8.07$ & $146.5 ; 174.9$ \\
\hline & $\mathrm{T} 2-4$ & & 81 & 0.067 & 1.97 & $0.94-4.12$ & \\
\hline & N0 & & 62 & 0.029 & 2.99 & $1.07-8.40$ & $145.4 ; 174.5$ \\
\hline & T2-4N0 & & 47 & 0.076 & 2.71 & $0.86-8.55$ & \\
\hline & T2-4N1-2 & & 34 & 0.596 & & & \\
\hline & Gastrointestinal, all & $\mathbf{b}$ & 99 & 0.009 & 2.51 & $1.24-5.09$ & $132.1 ; 161.9$ \\
\hline & Stage III & & 31 & 0.063 & 2.86 & $0.90-9.09$ & \\
\hline & $\mathrm{T} 2-4$ & & 81 & 0.034 & 2.18 & $1.04-4.57$ & $127.8 ; 156.4$ \\
\hline & N0 & & 62 & 0.263 & & & \\
\hline & N1-2 & & 37 & 0.013 & 3.87 & $1.23-12.23$ & $87.9 ; 154.4$ \\
\hline & G1-2 & & 54 & 0.020 & 2.84 & $1.13-7.11$ & $121.7 ; 160.4$ \\
\hline & T2-4N0 & & 47 & 0.524 & & & \\
\hline & $\mathrm{T} 2-4 \mathrm{~N} 1-2$ & & 34 & 0.040 & 3.14 & $0.10-9.93$ & \\
\hline & Gastric, all & $\mathbf{a}$ & 62 & 0.170 & & & \\
\hline & Stages I-II & & 44 & 0.010 & 5.97 & $1.26-28.23$ & $133.7 ; 182.5$ \\
\hline & No & & 38 & 0.014 & 5.77 & $1.19-27.89$ & $134.6 ; 181.7$ \\
\hline & Gastric, all & $\mathbf{b}$ & 62 & 0.075 & 2.19 & $0.90-5.31$ & $134.1 ; 158.5$ \\
\hline & N1-2 & & 24 & 0.015 & 4.27 & $1.22-15.00$ & $71.7 ; 145.1$ \\
\hline
\end{tabular}

* Responders vs weak responders.

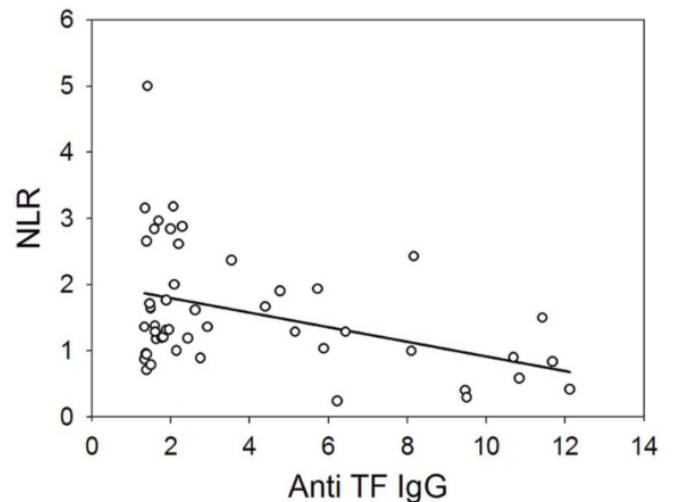

Fig 3. The correlation between the level of anti-TF IgG and neutrophil/lymphocyte ratio in patients with gastrointestinal cancer $(y=2.01$ $0.1(x)$.

\section{Discussion}

Natural anti-carbohydrate antibodies are mostly IgM-class antibodies. The study was focused on IgG antibodies because we observed a high anti-carbohydrate IgG in some long-term survivors [11, 13]. According to our preliminary data, no difference in survival was demonstrated in relation to anti-MUC1 IgM and anti-TF IgM antibodies [16]. The high level of anti-carbohydrate IgG and/or its postoperative elevation observed in some patients with cancer [13] may be a sign of an adaptive immune 
response which is indicative of the switching of antibodies to the IgG-class.

This study demonstrates that there is an association of the survival rate of patients with gastrointestinal cancer with the serum level of anti-carbohydrate IgG antibodies. The association of antibody levels with survival was revealed in the examination of patho-morphological subgroups. The increased preoperative or initial level of anti-TF and -Tn antibodies and the decreased level of anti- $\alpha \mathrm{Gal}$ antibodies were associated with a benefit in the survival rate (Tables 1 and 3, factor a). A significant association of the high preoperative level of anti-TF IgG with the survival of gastric cancer patients was demonstrated earlier [16]. However, in the present, longer follow-up of another, more homogeneous group of patients with gastric cancer, only a tendency to this association $(P=0.075$, Table 1) was noted. In the present study, we used the modified ELISA with an adequate negative control that made it possible to exclude from analysis serum samples with the level of anti-TF antibodies $<1$ (the results are presented in Table 1). A significantly better survival in TF-R vs TF-WR was observed in the gastrointestinal group and its subgroups, particularly in the G3 of gastrointestinal and gastric cancers. No association with survival in Tn-R vs Tn-WR and $\alpha$ Gal-R vs $\alpha \mathrm{Gal}-W \mathrm{R}$ in the whole gastric cancer group was found. Hamanaka $Y$ et al. established that there is no association of the serum anti- $\alpha \mathrm{Gal}$ IgG level with the survival of pancreatic cancer patients [17]. The preoperative level of anti-TF, $-\mathrm{Tn}$ or $-\alpha \mathrm{Gal}$ antibodies determined separately was of prognostic value for the groups examined. The combination of anti-TF and $-\alpha \mathrm{Gal}$ antibodies was more predictive as shown for the whole gastrointestinal group. The difference in survival between TF-R/ $\alpha$ Gal-WR and TF-WR/ $\alpha$ Gal-R was more significant than that between TF-R and TF-WR, or between $\alpha$ Gal-R and $\alpha$ Gal-WR.

To analyse the relation of antibodies level dynamics to survival, we used factors calculated as coefficients of a quadratic function. The study of the dynamics of anti-TF antibodies level did not reveal any additional groups to exist with different survival rates, but such groups were revealed while investigating the level of anti-Tn antibodies, namely, in stages I-II, T2-4 and N0, including colorectal cancer (factor a), as well as in stage III and N1-2 (gastric cancer, factor $\mathbf{b})$. Signifiant differences in survival rate between patients were revealed in the whole gastrointestinal group, as well as in groups T2-4, N1-2 and G1-2 when anti- $\alpha$ Gal antibodies were assessed by factor $b$. These results show that dynamic investigations were more informative for anti-Tn and $-\alpha$ Gal antibodies, being less informative for anti-TF antibodies probably due to inadequate time intervals of observations. It is noteworthy that survival in many groups of $\mathrm{R}$ vs WR differed significantly after a 10 -year observation as evaluated by the preoperative level of antibodies or their dynamics.

The lower level of preoperative anti-TF IgG dominated in stages III-IV or in metastases N1-2, as well as in patients with poorly differentiated carcinoma G3 [18]. The present study demonstrated that the lower preoperative level of anti-TF IgG (TF-WR) in the examined subgroups (Stage III, N1-2 and G3) is associated with worse survival. The postoperative level of anti-TF, $-\mathrm{Tn}$ and $-\alpha \mathrm{Gal}$ antibodies in patients with the G3 tumor of the gastrointestinal tract had increased to a different degree [13]. However, this positive dynamics was not associated with survival. The elevation of anti- $\alpha \mathrm{Gal}$ antibodies level was associated with poorer clinical outcome in many subgroups.

An absolute lymphocyte count is a surrogate marker of host immunity and is indicative of a better clinical outcome of patients with cancer [19]. NLR has been found to be indicative of systemic inflammation and stress. Moreover, the high values of NLR are a poor prognostic factor in oncological patients [20,21]. The level of anti-TF IgG directly correlated with the count of lymphocytes that may reflect the adaptive immune response but inversely correlated with the count of neutrophils and NLR that may be indicative of the noninvolvement of anti-TF antibodies in the inflammatory response (Figure 3). A significantly worse survival was demonstrated in $\alpha \mathrm{Gal}-\mathrm{R}$ having an elevated mean NLR. The combined determination of the level of anti- $\alpha \mathrm{Gal}$ antibodies and estimation of NLR was of prognostic value. The direct correlation between the level of anti- $\alpha$ Gal antibodies and NLR was revealed only in a small group of patients whose survival was worse. The anti- $\alpha \mathrm{Gal}$ immune response in some patients may be associated with an inflammation unfavourable in cancer. Inflammatory diseases (enterocolitis, pancreatitis and cholecystitis), postoperative ileus and infectious complications (suppuration of wound and abscess) were revealed in approximately $20 \%$ of $\alpha \mathrm{Gal}-\mathrm{R}$ and $\alpha \mathrm{Gal}-\mathrm{WR}$.

The association of survival with antibody level may be explained differently. An increased level of anti-TF antibodies in the circulation may be beneficial for cancer patients if antibodies could compete with circulating galectins for the binding to cancer-associated TF structure and thereby reduce the metastasis-promoting action of circulating galectins [22]. On the other hand, the serum level of galectins is greatly increased in patients with cancer and a high level of galectin 3 (the major known TF-binding protein) is associated with the worse survival of patients $[22,23]$. In ELISA, the increased level of galectin 3 in 
serum samples may compete with anti-TF antibodies for the binding of TF-conjugate, thereby influencing the results of determination of anti-TF IgG. Both suppositions need further detailed investigations.

Many anti-carbohydrate antibodies are cross-reactive to histo-blood group related antigens that may be aberrantly expressed in tumors as precursors or incompatible antigens [15, 24]. In this respect, some genera of enteric microbiota stimulating TACA-cross-reactive antibodies might be beneficial in cancer patients. We attempted to characterize the specificity of antibodies, and isolated anti-TF and anti-Tn IgGs from the serum samples of patients with gastrointestinal cancer, using PAA glycoconjugate-sorbents. The antibodies display a low specificity to tumor-associated and tumor-derived mucins [25]. In the indirect and competitive ELISA, the anti-TF IgG antibodies were more reactive to TF $\beta$ (Gal $\beta 1-3 G a l N A c \beta)$ than to TF (TFa) conjugates, and they were a heterogenic population varying in the cross-reactivity to GA1 and other glycans [25-27]. The populations of autoantibodies cross-reactive to TF $\beta$ and GA1 have been characterized in healthy donors and neurological patients [28-30]. The anti-TF IgG antibodies examined in the present study are unrelated to neuropathies rather because neurological disorders were observed in neither cancer patients nor donors having a high anti-TF IgG level.

Gastrointestinal tumors develop in the environment of commensal and pathogenic microbiota. Anti-TF antibodies may be induced by the TF-related antigens of enteric bacteria. The protein bands were immunostained on the blots of $H$. pylori extracts, using TF-specific monoclonal antibodies [31]. However, structures, which are immunochemically identical to TFa, are extremely rare on the surface of human intestinal bacteria [32]. The Tn antigen is expressed in glycans of parasites and related to different disorders and diseases [33]. The anti- $\alpha \mathrm{Gal}$ antibodies demonstrate the cross-reactivity to $\alpha \mathrm{Gal} \mathrm{mimic} \mathrm{or}$ blood group related glycans $[15,34,35]$. The anti- $\alpha \mathrm{Gal}$ antibodies were found to interact with a variety of enterobacteria and are possibly implicated in inflammatory processes due to the binding of bacteria adhered to human cells [36]. The level of anti- $\alpha \mathrm{Gal}$ $\operatorname{IgG}$ in benign gastrointestinal diseases, including Crohn's disease, has significantly increased [16, 37]. The increased level of antibodies in benign diseases may be related to bacterial translocation (BT). BT frequently occurs in patients with benign and malignant gastrointestinal diseases $[2,3]$ and may be one of the reason of an augmented immune response. In colorectal cancer patients, BT in the mesenteric lymph nodes is indicative of a worse outcome [38]. In fact, BT identified in patients with colorectal cancer is increased in deep invasion (T3) [3]. We demonstrated that the survival of $\alpha \mathrm{Gal}-\mathrm{R}$ vs $\alpha \mathrm{Gal}-\mathrm{WR}$ in the gastrointestinal (T2-T4) and colorectal cancer groups was worse (Table 1) and, as shown earlier, the level of anti- $\alpha$ Gal IgG was significantly higher in patients with tumor size T2-T3 vs T1 [18].

Neoplasia and inflammation can disrupt the intact mucosal barrier to intraluminal bacteria and bacterial antigens. An inflammation promotes the invasion and metastasis of tumor cells [39]. A striking difference in microbial colonization patterns between tumor tissue and adjacent non-malignant mucosa or control tissues has been found $[1,40]$. The colonization may cause apoptosis in tumors or, on the contrary, promote the tumor progression [41-43] as well as distort the anti-tumor immune response. We suppose that the level of anti-TF, $-\mathrm{Tn}$ and $-\alpha \mathrm{Gal}$ IgGs reflects the immune response to microorganisms and may be indirectly associated with better or worse survival via beneficial or detrimental interrelation between enteric microorganisms and tumor.

In conclusion, the level of serum anti-TF, -Tn and $-\alpha \mathrm{Gal}$ antibodies was associated with survival of patients with gastrointestinal cancer in a long-term investigation. In different patho-morphological subgroups, the preoperative levels of antibodies and their dynamics was found to be of prognostic significance. Patients with elevated levels of anti-TF and -Tn antibodies showed a longer survival time while, on the contrary, the survival time was shorter in patients with an elevated level of anti- $\alpha \mathrm{Gal}$ antibodies. The simple method for determination of serum antibodies level may be a useful supplement in clinical outcome assessment.

\section{Abbreviations}

TF: Thomsen-Friedenreich antigen; Tn: GalNAca; PAA: polyacrylamide; NLR: neutrophil/ lymphocyte ratio; TACA: tumor-associated carbohydrate antigens; R: responders; WR: weak responders; BT: bacterial translocation.

\section{Acknowledgements}

We are grateful to Dr. A. Lipping for providing histological data, and to R. Syld for correcting English.

\section{Grant}

This study was supported by a grant \# 8399 from the Estonian Science Foundation.

\section{Authorship}

ES designed and performed research, analyzed and interpreted data, and wrote the manuscript. BS and $\mathrm{KK}$ performed calculations and statistical analysis. VC analyzed data. OK designed research, 
interpreted data and revised the manuscript. All authors read, gave comments, and approved the final version of the manuscript.

\section{Conflict of Interest}

The authors have declared that no conflict of interest exists.

\section{References}

1. Marchesi JR, Dutilh BE, Hall N, et al. Towards the human colorectal cancer microbiome. PLoS One. 2011; 6: e20447.

2. MacFie J, Reddy BS, Gatt M, et al. Bacterial translocation studied in 927 patients over 13 years. Br J Surg. 2006; 93: 87-93.

3. Takesue $\mathrm{Y}$, Kakehashi M, Ohge H, et al. Bacterial translocation: not a clinically relevant phenomenon in colorectal cancer. World J Surg. 2005; 29: 198-202.

4. Springer GF. Immunoreactive $\mathrm{T}$ and Tn epitopes in cancer diagnosis, prognosis, and immunotherapy. J Mol Med. 1997; 75: 594-602.

5. Campbell BJ, Finnie IA, Hounsell EF, et al. Direct demonstration of increased expression of Thomsen-Friedenreich (TF) antigen in colonic adenocarcinoma and ulcerative colitis mucin and its concealment in normal mucin. J Clin Invest. 1995; 95: 571-6.

6. Yu LG. The oncofetal Thomsen-Friedenreich carbohydrate antigen in cancer progression. Glycoconj J. 2007; 24: 411-20.

7. Galili U. Evolution and pathophysiology of the human natural anti-alpha-galactosyl IgG (anti-Gal) antibody. Springer Semin Immunopathol. 1993; 15: 155-71.

8. Springer GF, Tegtmeyer H. Origin of anti-Thomsen-Friedenreich (T) and Tn agglutinins in man and in white Leghorn chicks. Br J Haematol. 1981; 47: 453-60.

9. Bovin NV. Polyacrylamide-based glycoconjugates as tools in glycobiology. Glycoconj J. 1998; 15: 431-46.

10. Smorodin JP, Kurtenkov OA, Miljukhina LM, et al. Thomsen-Friedenreich antigen-specific IgM antibodies: diagnostic significance for gastric and breast cancer. Exp Oncol. 1997; 19: 338-42.

11. Smorodin EP, Kurtenkov OA, Sergeyev BL, et al. Antibodies to tumor-associated carbohydrate epitopes in sera of cancer patients and blood donors. Exp Oncol. 2001; 23(2): 109-13.

12. Desai PR, Ujjainwala LH, Carlstedt SC, et al. Anti-Thomsen-Friedenreich (T) antibody-based ELISA and its application to human breast carcinoma detection. J Immunol Meth. 1995; 188: 175-85.

13. Smorodin EP, Kurtenkov OA, Sergeyev BL, et al. Postoperative change of anti-Thomsen-Friedenreich and Tn IgG level: the follow-up study of gastrointestinal cancer patients. World J Gastroenterol. 2008; 14: 4352-8.

14. Sobin LH, Gospodarowicz MK, Wittekind C. TNM Classification of Malignant Tumours, 7th Edition. Wiley-Blackwell. 2009.

15. Smorodin EP, Kurtenkov OA, Sergeyev BL, et al. Specificity of serum anti-A(di) IgG antibodies from patients with gastrointestinal cancer. J Immunoassay Immunochem. 2011; 32: 170-90.

16. Kurtenkov O, Klaamas K, Mensdorff-Pouilly S, et al. Humoral immune response to MUC1 and to the Thomsen-Friedenreich (TF) glycotope in patients with gastric cancer: Relation to survival. Acta Oncol. 2007; 46: 316-23.

17. Hamanaka Y, Suehiro Y, Fukui M, et al. Circulating anti-MUC1 IgG antibodies as a favorable prognostic factor for pancreatic cancer. Int J Cancer. 2003; 103: 97-100.

18. Smorodin EP, Kurtenkov OA, Sergeyev BL, et al. The relation of serum anti-TF, Tn and alpha-Gal IgG antibody levels to cancer progression and histopathological grading. Exp Oncol. 2002; 24(4): 270-3.

19. Porrata LF, Markovic SN. Is absolute lymphocyte count just another prognostic factor in cancer? SRX Medicine. 2010; 2010: 1-8.

20. Zahorec R. Ratio of neutrophil to lymphocyte counts - rapid and simple parameter of systemic inflammation and stress in critically ill. Bratisl Lek Listy. 2001; 102: 5-14.

21. Walsh SR, Cook EJ, Goulder F, et al. Neutrophil-lymphocyte ratio as a prognostic factor in colorectal cancer. J Surg Oncol. 2005; 91: 181-4.

22. Barrow $\mathrm{H}$, Guo $\mathrm{X}$, Wandall $\mathrm{HH}$, et al. Serum galectin-2, -4 , and -8 are greatly increased in colon and breast cancer patients and promote cancer cell adhesion to blood vascular endothelium. Clin Cancer Res. 2011; 17: 7035-46.

23. Barrow H, Rhodes JM, Yu LG. Simultaneous determination of serum galectin-3 and -4 levels detects metastases in colorectal cancer patients. Cell Oncol (Dordr). 2013; 36: 9-13.

24. Hakomori S. Antigen structure and genetic basis of histo-blood groups A, B and $\mathrm{O}$ : their changes associated with human cancer. Biochim Biophys Acta. 1999: 1473: 247-66.

25. Smorodin EP, Kurtenkov OA, Sergeyev BL, et al. Specificity of human anti-carbohydrate IgG antibodies as probed with polyacrylamide-based glycoconjugates. Glycoconj J. 2004; 20: 83-9.

26. Smorodin EP, Kurtenkov OA, Sergeyev BL, et al. The Characterization of Cross-reactive Antibodies to Thomsen-Friedenreich $\alpha / \beta$ and Related Glycan-conjugates with Polyacrylamide Carriers in Patients with Gastrointestinal Cancer. J Clin Cell Immunol. 2011; S5.
27. Butschak $G$, Karsten $U$. Isolation and characterization of Thomsen-Friedenreich-specific antibodies from human serum. Tumor Biol. 2002; 23: 113-22.

28. Mizutamari RK, Wiegandt H, Nores GA. Characterization of anti-ganglioside antibodies present in normal human plasma. J Neuroimmunol. 1994; 50: 215-20.

29. Lopez PH, Lardone RD, Irazoqui FJ, et al. The origin of anti-GM1 antibodies in neuropathies: the "binding site drift" hypothesis. Neurochem Res. 2002; 27: 687-95.

30. Lopez PH, Comín R, Villa AM, et al. A new type of anti-ganglioside antibodies present in neurological patients. Biochim Biophys Acta. 2006; 1762: 357-61.

31. Klaamas K, Kurtenkov O, Rittenhouse-Olson K, et al. Expression of tumor-associated Thomsen-Friedenreich antigen ( $\mathrm{T} \mathrm{Ag}$ ) in Helicobacter pylori and modulation of $\mathrm{T} \mathrm{Ag}$ specific immune response in infected individuals. Immunol Invest. 2002; 31: 191-204.

32. Henderson G, Ulsemer P, Schöber $U$, et al. Occurrence of the human tumor-specific antigen structure GalB1-3GalNAca- (Thomsen-Friedenreich) and related structures on gut bacteria: prevalence, immunochemical analysis and structural confirmation. Glycobiology. 2011; 21: 1277-89.

33. Ju T, Otto VI, Cummings RD. The Tn antigen-structural simplicity and biological complexity. Angew Chem Int Ed Engl. 2011; 50: 1770-91.

34. Teneberg SI, Lönnroth I,Torres Lopez JF, et al. Molecular mimicry in the recognition of glycosphingolipids by Gal alpha 3 Gal beta 4 GlcNAc beta-binding Clostridium difficile toxin A, human natural anti alpha-galactosyl IgG and the monoclonal antibody Gal-13: characterization of a binding-active human glycosphingolipid, non-identical with the animal receptor. Glycobiology. 1996; 6: 599-609.

35. Galili U. Xenotransplantation and $\mathrm{ABO}$ incompatible transplantation: the similarities they share. Transfus Apher Sci. 2006; 35: 45-58.

36. Galili U, Mandrell RE, Hamadeh RM, et al. Interaction between human natural anti-alpha-galactosyl immunoglobulin $\mathrm{G}$ and bacteria of the human flora. Infect Immun. 1988; 56: 1730-7.

37. D'Alessandro M, Mariani P, Lomanto D, et al. Alterations in serum anti-alpha-galactosyl antibodies in patients with Crohn's disease and ulcerative colitis. Clin Immunol. 2002; 103: 63-8.

38. Chin KF, Kallam R, O'Boyle C, et al. Bacterial translocation may influence the long-term survival in colorectal cancer patients. Dis Colon Rectum. 2007; 50; 323-30.

39. Solinas G, Marchesi F, Garlanda C, et al. Inflammation-mediated promotion of invasion and metastasis. Cancer Metastasis Rev. 2010; 29: 243-8.

40. Abdulamir AS, Hafidh RR, Bakar FA. Molecular detection, quantification, and isolation of Streptococcus gallolyticus bacteria colonizing colorectal tumors: inflammation-driven potential of carcinogenesis via IL-1, COX-2, and IL-8. Mol Cancer. 2010; 9: 249

41. Toley BJ, Forbes NS. Motility is critical for effective distribution and accumulation of bacteria in tumor tissue. Integr Biol. 2012; 4: 165-76.

42. Castellarin M, Warren RL, Freeman JD, et al. Fusobacterium nucleatum infection is prevalent in human colorectal carcinoma. Genome Res. 2012; 22: 299-306.

43. Huang B, Zhao J, Shen S, et al. Listeria monocytogenes promotes tumor growth via tumor cell toll-like receptor 2 signaling. Cancer Res. 2007; 67: 4346-52. 